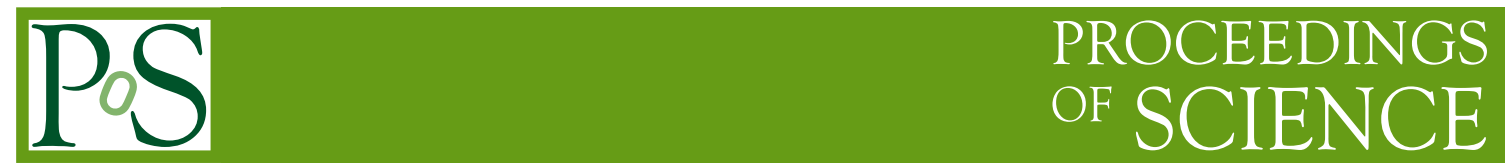

\title{
Component Separation in Polarization with FastICA
}

\section{Federico Stivoli*}

SISSA/ISAS

E-mail: stivoliesissa.it

FastICA is a blind technique aimed to separate different components in CMB experiments, with a very few assumptions on the signals to recover. Since current knowledge about foregrounds in polarization are very poor, this kind of technique can play a crucial role in forecoming CMB experiments. Recent and ongoing developments of the method are presented here

$C M B$ and Physics of the Early Universe 20-22 April 2006

Ischia, Italy

${ }^{*}$ Speaker. 


\section{The Component Separation Problem}

In any Cosmic Microwave Background experiment, it is unavoidable to detect contributions from any foreground between us and the last scattering surface, added to the cosmological signal. Basically what we actually measure is a mixture $x$ of different components $s_{j}$, mixed up by a matrix $A$, that takes into account the different frequency scalings of the different components.

$$
x_{i}=A_{i}^{j} s_{j}+n_{i}
$$

here the subscript $i$ indicates the frequency channels of the detectors and $n_{i}$ is the instrumental noise.

Now, the aim of any component separation technique is to recover the signals $s_{j}$. There are basically two diffent approaches to solve the problem in 1.1, depending on how reliable are assumptions on the mixing matrix. 'Non-blind' techniques exploit a priori knowledges about the signal to recover and try to estimate parameters that define their spectral behaviour. These algorithm proved to work very well in the past, e.g. the maximum entropy method $[8,14]$ and Wiener filtering [15, 3], but they have an evident drawback in the fact that if the assumptions are not correct, provided results will be biased. Then, in the case of CMB polarization experiment, since the polarized foregrounds are likely to be greatly uncertain, alternative approaches, not relying on such priors, may be required. These second class of component separation techniques is called 'blind'. They only exploit the statistical independence of the sky signals to be separated, a natural expectation for the CMB and Galactic emissions. Within this class there is the Independent Component Analysis (ICA) technique.

\section{Independent Component Analysis (ICA)}

The core of the ICA principle consists in the maximization of the neg-entropy, which measures the distance of a mixture of signals from a Gaussian distribution [1,7]. The hypothesis are that the mixture contains at most one Gaussian component and that all of them obey different probability distributions and frequency scalings; under these assumptions it is possible, exploiting the central limit theorem, to demonstrate that each local maximum of the neg-entropy corresponds to one of the component present into the mixture. FastICA is an application of this principle.

\subsection{Major Achievements}

FastICA has been successfully tested on the real data from COBE/DMR [10], recovering the main CMB results of that experiments, concerning the amplitude and power spectrum of the cosmological perturbations on large scales and also on the data of the BEAST experiment [6]. It also provided good results when applied to simulated Planck data both in total intensity [9] and polarization on all sky [2]. Finally, as we show in the next section this blind component separation technique proved to have the capability to recover the $B$ modes of the $\mathrm{CMB}$, on a limited patch of the sky [13]. 


\subsection{A successful example}

In the next future, many experiments will try a detection of CMB $B$ modes on patch of the sky [11], [4]. That is a real challenge from a practical point of view, for the following reasons. First of all, we still don't know the actual level of the cosmological tensor contribution, since theory is not albe to lead us in this aspect. Then, even in the best situation, the signal would be extremely low and new technologies (e.g. large array of detectors) must be used to detect it buried in the noise and to keep systematic effect under control as much as possible. Finally, simulations and data from the third year release of WMAP [12] clearly show that foregrounds are dominating the polarized sky on large scales at all frequencies of cosmological interest. This means that some kind of component separation must be performed in any attempt of $B$ modes detection.

For these reason we tested FastICA in a simulated environment close to those expected from the next sub orbital CMB experiments, on a limited patch of the sky and in the presence of a substantial foreground contamination as estimated on the basis of the current models of the Galactic emission.

We consider two fiducial observations, one operating at low $(40,90 \mathrm{GHz})$ and one at high $(150,350 \mathrm{GHz})$ frequencies and thus dominated by the synchrotron and thermal dust emission, respectively. We use a parallel version of the FastICA code to explore a substantial parameter space including Gaussian pixel noise level, observed sky area and the amplitude of the foreground emission and employ large Monte Carlo simulations to quantify errors and biases pertinent to the reconstruction for different choices of the parameter values. We identify a large subspace of the parameter space (even in the cases when the $B$ mode CMB signal is up to a few times weaker than the foreground contamination and the noise amplitude is comparable with the total CMB polarized emission.) for which the quality of the CMB reconstruction is excellent, i.e., where the errors and biases introduced by the separation are found to be comparable or lower than the uncertainty due to the cosmic variance and instrumental noise. In figure 1, we show the pseudo-B modes [5], extracted at $40 \mathrm{GHz}$ with a signal to noise ratio equal to 2 .

\section{Ongoing Developments}

Even if the algorithm proved to work properly in different environments, there are still tests to perform and developments to implement to optimize the tool for future experiments. Then it is important to become more and more realistic with the simulations. For this reason, in the next step we plan to take into account also the most common systematic effects $(1 / f$ noise, asymmetric beam and so on) and to add the point sources contribution in the simulated skies.

On the other hand, it is possible to improve the code itself. For example, we are studying how to implement a flexible version of the algorithm, keeping the possibility to impose very well known priors in the separation process, getting closer to a semi-blind approach.

Finally, in the view of working with the Planck data, we are working on a new parallel version of the code. Because of the high resolution and the large number of channels of the Planck experiment, it will be necessary to spread the separation on many processors in order to avoid memory issues. For this reason a fully parallelized version of the very core of the algorithm is being implemented. 


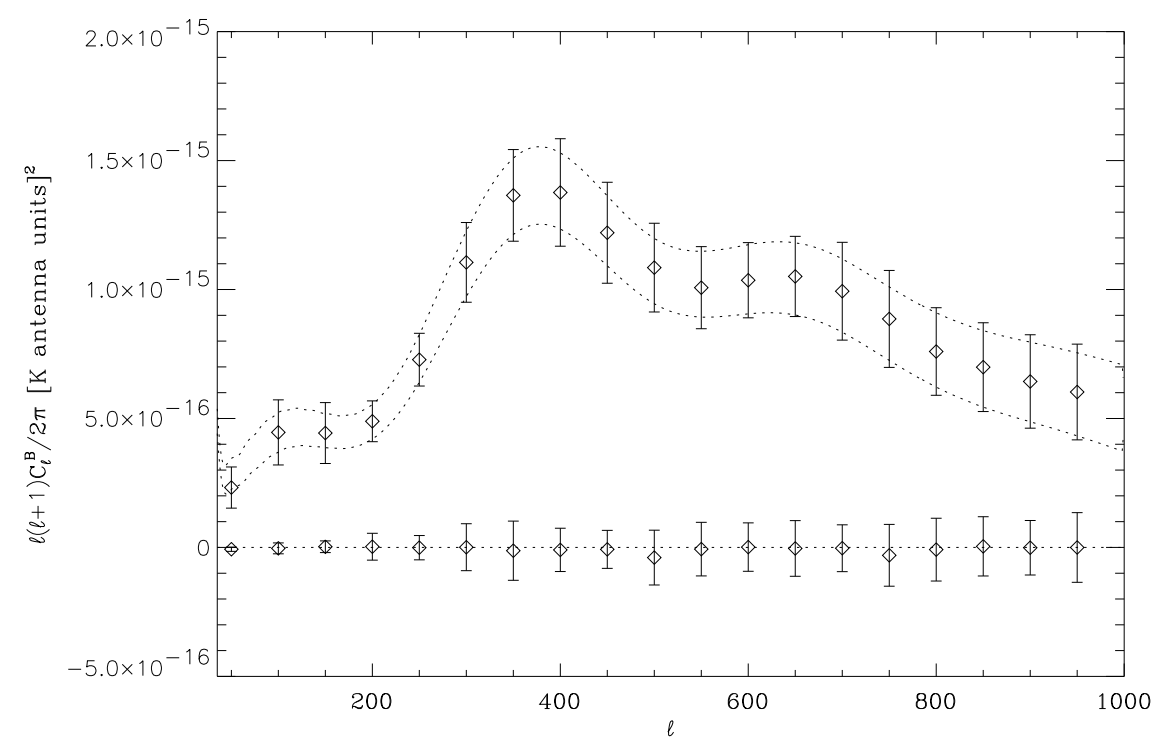

Figure 1: Power spectra of the reconstructed $\tilde{C}_{l}^{B}$ modes of the CMB at $40 \mathrm{GHz}$. The region between the dotted lines is the theoretical CMB signal $\pm \sigma$ cosmic and noise variance on the sky area considered. At the bottom we show the average and standard deviation of the residuals on each realization.

\section{References}

[1] Amari S., Chichocki A., 1998, Proc. IEEE 86, 2026

[2] Baccigalupi C. et al., 2004, MNRAS 355, 5570

[3] Bouchet F.R., Prunet S., Sethi S.K., 1999, MNRAS , 302, 663

[4] Bowden M. et al., 2004, MNRAS 349, 321

[5] Chon, G., Challinor A., Prunet S., Hivon E. Szapudi I., 2004, MNRAS 350, 914

[6] Donzelli S. et al., 2005, MNRAS in press, preprint arxiv.org/abs/astro-ph/0507267

[7] Hyvärinen A., 1999, IEEE Signal Processing Lett. 6, 145

[8] Hobson M.P., Jones A.W., Lasenby A.N., Bouchet F., 1998, MNRAS 300, 1

[9] Maino D. et al., 2002, MNRAS 334, 53

[10] Maino D., Banday A.J., Baccigalupi C., Perrotta F., Górski K., 2003, MNRAS 344, 544

[11] Oxley P. et al., 2004, Earth Observing Systems IX. Edited by William L. Barnes and James J. Butler, Proceedings of the SPIE, 5543, 320

[12] Page L. et al., 2006, preprint arxiv.org/abs/astro-ph/0603450

[13] Stivoli F. et al., 2005, MNRAS submitted, preprint arxiv.org/abs/astro-ph/0505381

[14] Stolyarov V., Hobson M.P., Ashdown M.A.J., Lasenby A.N., 2002, MNRAS 336, 97

[15] Tegmark M., Efstathiou G., 1996, MNRAS 281, 1297 